\title{
Nuclear grade and necrosis predict prognosis in malignant epithelioid pleural mesothelioma: a multi-institutional study
}

Lauren E Rosen ${ }^{1}$, Theodore Karrison ${ }^{2}$, Vijayalakshmi Ananthanarayanan ${ }^{3}$, Alexander J Gallan ${ }^{1}$, Prasad S Adusumilli ${ }^{4}$, Fouad S Alchami ${ }^{5}$, Richard Attanoos ${ }^{6}$, Luka Brcic $^{7}$, Kelly J Butnor ${ }^{8}$, Françoise Galateau-Sallé ${ }^{9}$, Kenzo Hiroshima ${ }^{10}$, Kyuichi Kadota ${ }^{11}$, Astero Klampatsa ${ }^{12}$, Nolween Le Stang ${ }^{9}$, Joerg Lindenmann ${ }^{13}$, Leslie A Litzky ${ }^{14}$, Alberto Marchevsky ${ }^{15}$, Filomena Medeiros ${ }^{16}$, M Angeles Montero ${ }^{17}$, David A Moore ${ }^{18}$, Kazuki Nabeshima ${ }^{19}$, Elizabeth N Pavlisko ${ }^{20}$, Victor L Roggli ${ }^{20}$, Jennifer L Sauter ${ }^{21}$, Anupama Sharma ${ }^{22}$, Michael Sheaff ${ }^{23}$, William D Travis ${ }^{21}$, Wickii T Vigneswaran ${ }^{24}$, Bart Vrugt ${ }^{25}$, Ann E Walts ${ }^{15}$, Melissa Y Tjota ${ }^{1}$, Thomas Krausz ${ }^{1}$ and Aliya N Husain ${ }^{1}$

${ }^{1}$ Department of Pathology, The University of Chicago Medicine, Chicago, IL, USA; ${ }^{2}$ Department of Public Health Sciences, The University of Chicago, Chicago, IL, USA; ${ }^{3}$ Department of Pathology, Loyola University Medical Center, Chicago, IL, USA; ${ }^{4}$ Department of Thoracic Surgery, Memorial Sloan Kettering Cancer Center, New York, NY, USA; ${ }^{5}$ Department of Cellular Pathology, University Hospital of Wales, Cardiff, UK;

${ }^{6}$ Department of Cellular Pathology, University Hospital of Wales and Cardiff University, Cardiff, UK; ${ }^{7}$ Medical University of Graz, Institute of Pathology, Graz, Austria; ${ }^{8}$ Department of Pathology, University of Vermont Medical Center, Burlington, VT, USA; ${ }^{9}$ Centre Léon Bérard, BioPathologie, Lyon, France; ${ }^{10}$ Department of Pathology, Tokyo Women's Medical University Yachiyo Medical Center, Yachiyo, Japan; ${ }^{11}$ Department of Diagnostic Pathology, Kagawa University, Takamatsu, Japan; ${ }^{12}$ Division of Pulmonary, Allergy and Critical Care, University of Pennsylvania Perelman School of Medicine, Philadelphia, PA, USA; ${ }^{13}$ Division of Thoracic and Hyperbaric Surgery, Department of Surgery, Medical University of Graz, Graz, Austria; ${ }^{14}$,Department of Pathology, University of Pennsylvania Perelman School of Medicine, Philadelphia, PA, USA; ${ }^{15}$ Department of Pathology \& Laboratory Medicine, Cedars-Sinai Medical Center, Los Angeles, CA, USA; ${ }^{16}$ Basildon \& Thurrock University Hospital, Department of Pathology, Basildon, UK; ${ }^{17}$ Department of Histopathology, Royal Brompton and Harefield Hospitals Imperial College of London, London, UK; ${ }^{18}$ Department of Cancer Studies, University of Leicester, Leicester, UK; ${ }^{19}$ Fukuoka University, Department of Pathology, Fukuoka, Japan; ${ }^{20}$ Department of Pathology, Duke University Medical Center, Durham, NC, USA; ${ }^{21}$ Department of Pathology, Memorial Sloan Kettering Cancer Center, New York, NY, USA; ${ }^{22}$ Department of Pathology, University of Pittsburgh Medical Center and VA Pittsburgh Healthcare System, Pittsburgh, PA, USA; ${ }^{23}$ Department of Pathology, Barts Health NHS Trust, London, UK; ${ }^{24}$ Department of Thoracic and Cardiovascular Surgery, Loyola University Medical Center, Chicago, IL, USA and ${ }^{25}$ University Hospital Zurich, Institute for Pathology and Molecular Pathology, Zurich, Switzerland

A recently described nuclear grading system predicted survival in patients with epithelioid malignant pleural
mesothelioma. The current study was undertaken to validate the grading system and to identify additional
prognostic factors. We analyzed cases of epithelioid malignant pleural mesothelioma from 17 institutions across
the globe from 1998 to 2014 . Nuclear grade was computed combining nuclear atypia and mitotic count into a
grade of I-III using the published system. Nuclear grade was assessed by one pathologist for three institutions,
the remaining were scored independently. The presence or absence of necrosis and predominant growth pattern
were also evaluated. Two additional scoring systems were evaluated, one combining nuclear grade and necrosis
and the other mitotic count and necrosis. Median overall survival was the primary endpoint. A total of 776 cases

Correspondence: Dr AN Husain, MBBS, Department of Pathology, The University of Chicago, 5841 S. Maryland Avenue, MC6101, Chicago, IL 60637, USA.

E-mail: Aliya.Husain@uchospitals.edu

Received 20 July 2017; revised 15 October 2017; accepted 16 October 2017; published online 12 January 2018 
were identified including 301 (39\%) nuclear grade I tumors, 354 (45\%) grade II tumors and 121 (16\%) grade III tumors. The overall survival was 16 months, and correlated independently with age $(P=0.006)$, sex $(0.015)$, necrosis $(0.030)$, mitotic count $(0.001)$, nuclear atypia $(0.009)$, nuclear grade $(<0.0001)$, and mitosis and necrosis score $(<\mathbf{0 . 0 0 0 1 )}$. The addition of necrosis to nuclear grade further stratified overall survival, allowing classification of epithelioid malignant pleural mesothelioma into four distinct prognostic groups: nuclear grade I tumors without necrosis (29 months), nuclear grade I tumors with necrosis and grade II tumors without necrosis (16 months), nuclear grade II tumors with necrosis (10 months) and nuclear grade III tumors (8 months). The mitosis-necrosis score stratified patients by survival, but not as well as the combination of necrosis and nuclear grade. This study confirms that nuclear grade predicts survival in epithelioid malignant pleural mesothelioma, identifies necrosis as factor that further stratifies overall survival, and validates the grading system across multiple institutions and among both biopsy and resection specimens. An alternative scoring system, the mitosis-necrosis score is also proposed.

Modern Pathology (2018) 31, 598-606; doi:10.1038/modpathol.2017.170; published online 12 January 2018

The most common primary malignant tumor of the pleura is malignant mesothelioma, which shows a strong association with asbestos exposure. ${ }^{1}$ Malignant pleural mesotheliomas are aggressive tumors with a median survival of 9-12 months despite multimodality therapy. ${ }^{1-4}$ Owing to the poor outcome, much effort has been made to define prognostic factors that accurately stratify patients for therapy. It is well established that tumors with epithelioid histology have a more favorable prognosis compared to sarcomatoid and biphasic types. ${ }^{2,3}$ Histology is the most important prognostic variable that, together with TNM staging, forms the basis for treatment decisions. ${ }^{3,5}$

A group from Memorial Sloan Kettering Cancer Center sought to identify additional pathologic factors that predict prognosis in epithelioid malignant pleural mesothelioma. ${ }^{6,7}$ In addition to histologic subtyping, histologic grading was assessed in which seven nuclear features were evaluated, and while five correlated with the length of overall survival, only two, nuclear atypia and mitotic count, were found to be independent prognostic factors. ${ }^{6,7}$ These two features were used to create a three-tier nuclear grading system that proved itself an independent predictor of overall survival. ${ }^{6}$ The goals of this study are (1) to validate the Memorial Sloan Kettering Cancer Center nuclear grading system using a large number of cases from multiple institutions, (2) to evaluate the prognostic significance of additional histologic features including necrosis and growth pattern, and (3) to determine whether this system is still valid when nuclear grading is performed independently by pathologists from different institutions.

\section{Materials and methods}

Clinical and pathologic data on patients diagnosed with epithelioid malignant pleural mesothelioma between 1998 and 2014 was collected from 17 institutions worldwide. Institutional Review Board approvals from each institution were obtained for this study. Clinical variables collected included age, sex, date of diagnosis, surgical procedure, $\mathrm{T}$ stage, $\mathrm{N}$ status, and date of death or last follow-up. Median overall survival was measured in months since initial diagnosis.

Pathologic diagnosis was based on accepted histologic and immunohistochemical criteria. ${ }^{8,9}$ Histologic features were evaluated by one pathologist for three institutions (University of Chicago, Duke University and Tokyo Women's Medical University) and independently by pathologists from the remaining institutions. An average of three representative hematoxylin and eosin (H\&E)-stained slides were reviewed from each case (range 2-20). Nuclear atypia and mitotic count were evaluated as described previously by Kadota et al. ${ }^{6}$ Nuclear features were evaluated at $\times 400$. Mitoses were counted after identifying the areas of highest mitotic activity as an average of mitotic figures per 10 high power fields (HPF) (Figure 1a). Tumors were divided into three groups based on the mitotic count: low $0-1 / 10 \mathrm{HPF}$, intermediate $2-4 / 10 \mathrm{HPF}$, and high $\geq 5 / 10 \mathrm{HPF}$. The low, intermediate, and high groups were assigned mitotic count scores of 1,2 , and 3 respectively. Nuclear atypia was assessed in the area with the highest degree of atypia and was classified as mild atypia-uniform small nuclei with inconspicuous nucleoli, moderate atypia-nuclei intermediate in size with variability in shape and variably prominent nucleoli, and severe atypia-bizarre, enlarged nuclei, multinucleation, and macronucleoli in $>5 \%$ of tumor cells. Mild, moderate, and severe atypia were given nuclear atypia scores of 1, 2, and 3, respectively (Figure 2). A composite nuclear grade was assigned to each case upon addition of scores for nuclear atypia and mitotic count. Nuclear grade I was assigned to tumors with a score of $2-3$, grade II for scores $4-5$, and grade III for score 6 .

Necrosis was evaluated at $400 \times$ and scored as present or absent (Figure 1b). A mitosis-necrosis score was calculated for each case. Cases with five or more mitoses per $10 \mathrm{HPF}$ were given a score of 1 , and 


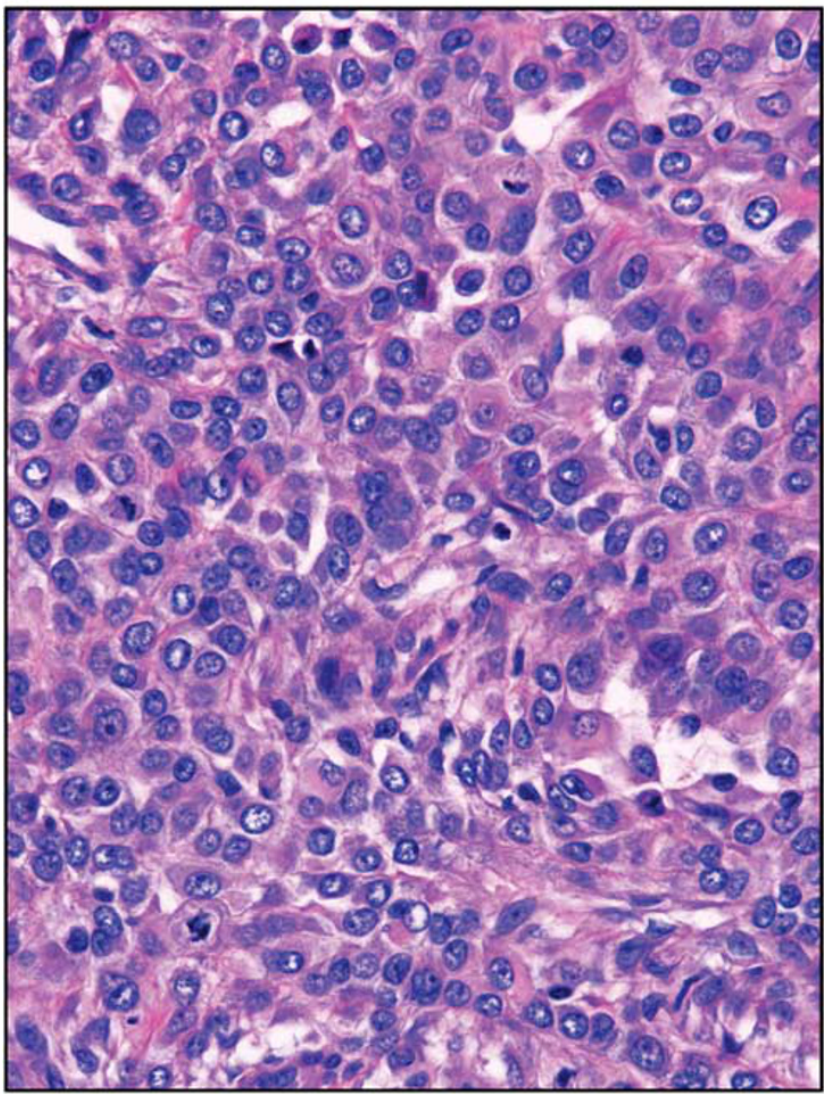

b

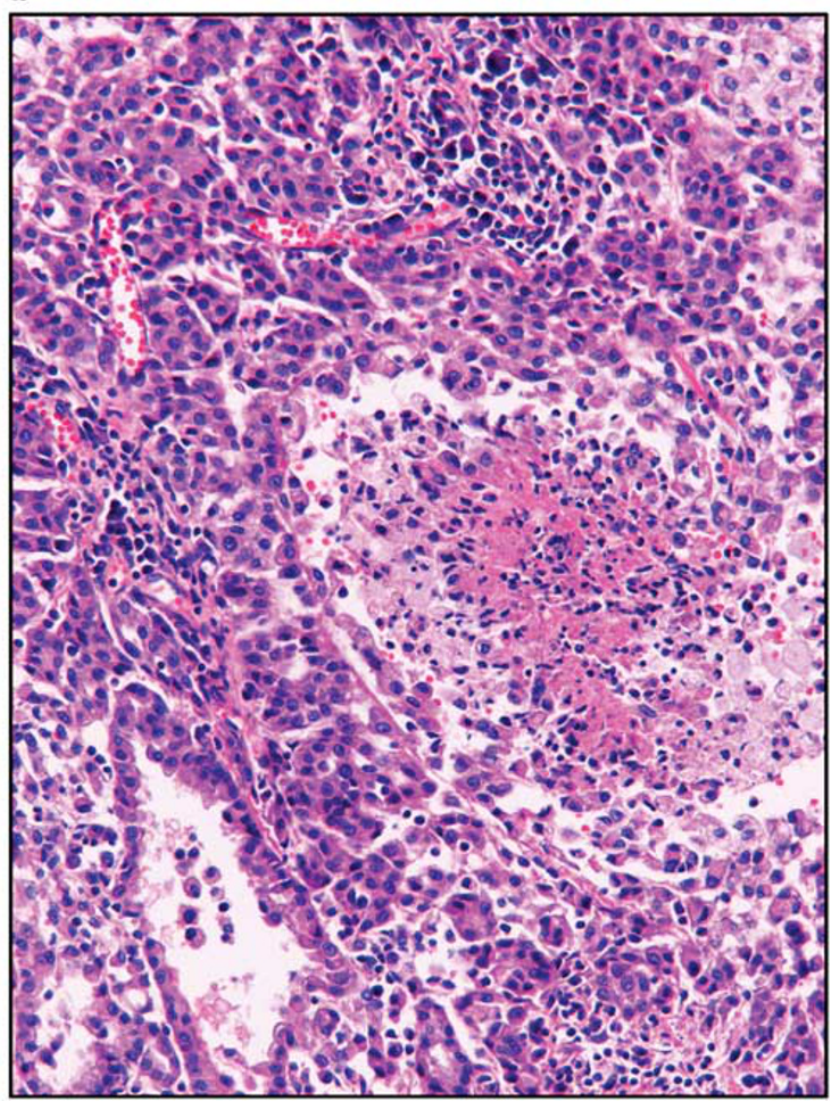

Figure 1 Histologic features of epithelioid diffuse malignant pleural mesothelioma (H\&E stain; original magnification, $\times 400$ : (a), $\times 200$ : (b)). (a) Nuclear grade I tumor with mild nuclear atypia and numerous mitotic figures. (b) Nuclear grade I tumor with necrosis.

a

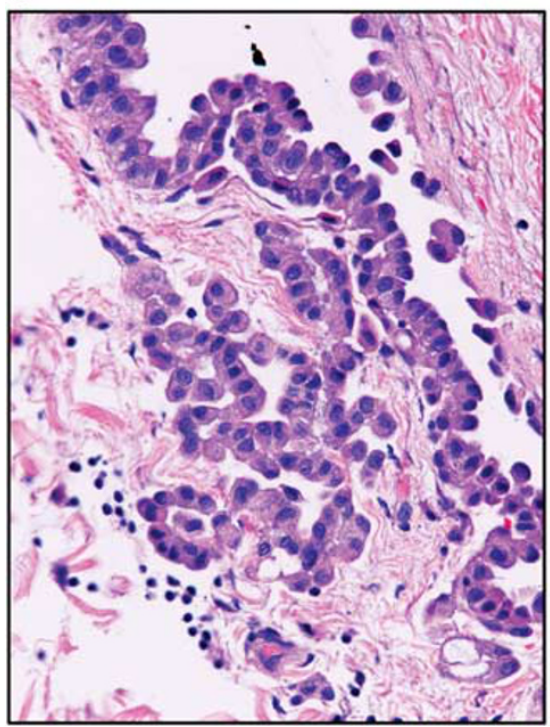

b

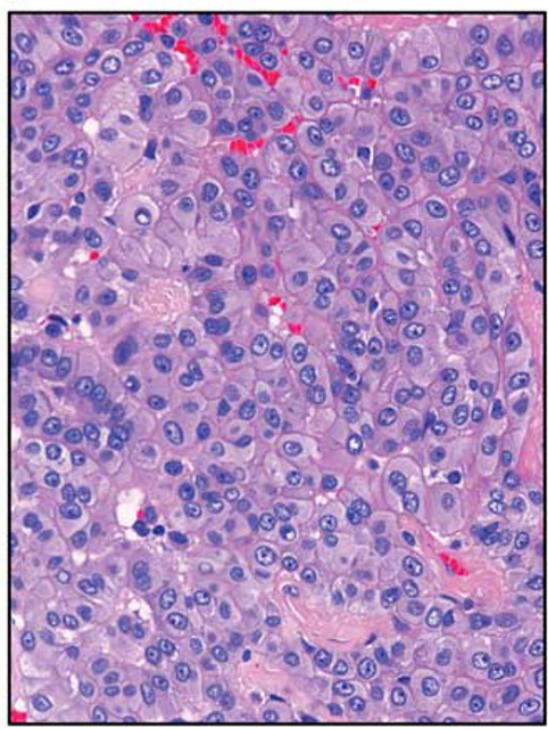

C

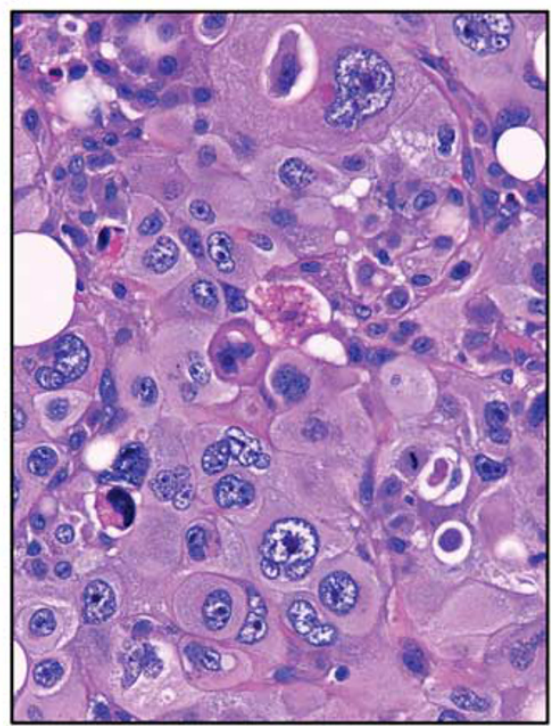

Figure 2 Nuclear atypia scoring of epithelioid diffuse malignant pleural mesothelioma (H\&E stain; original magnification, $\times 400$ : $(\mathbf{a}-\mathbf{c}))$. (a) Mild nuclear atypia with small bland nuclei and inconspicuous nucleoli. (b) Moderate nuclear atypia with mild variation is size and shape and conspicuous nucleoli. (c) Severe nuclear atypia with large pleomorphic nuclei. 
those with fewer were given a score of 0 . Necrosis was given a score of 1 if present and 0 if absent. These scores were then added to create the mitosisnecrosis score, ranging from 0 to II.

Histologic subtyping was performed according to the most recent (2015) World Health Organization criteria. ${ }^{8}$ A solid growth pattern in $\geq 50 \%$ of the tumor was recorded as predominately solid growth pattern. The solid pattern is defined as a tumor comprised of sheets and nests of round to polygonal cells without any other discrete architectural pattern. ${ }^{8}$

\section{Statistical Analysis}

Descriptive statistics (mean and standard deviation for continuous variables and frequency distributions for categorical data) are provided to summarize baseline demographic, clinical, and histological characteristics of the study cohort. T-tests and $\chi^{2}$ tests were performed to compare baseline variables between different groups of interest. Survival curves were estimated by the Kaplan-Meier method; median survival times and confidence intervals were generated from the Kaplan-Meier curves using the Brookmeyer-Crowley procedure. ${ }^{10,11}$ Survival curves were compared across different groups using the log-rank test, and univariate and multivariable Cox regression models were fit to assess and adjust for prognostic factors. ${ }^{12}$ Wald and likelihood ratio tests were performed to determine the statistical significance of potential prognostic factors, as well as interactions among the factors. The proportional hazards assumption was checked using log-log plots. ${ }^{13}$

\section{Results}

A total of 776 cases were identified, with an average of 46 cases from each institution (range 1-170) (Table 1). The mean age was 65 years (range 29-91) and the majority of patients were male $(611,79 \%)$ with a male to female ratio of $3.7: 1$. By procedure, 269 patients (35\%) underwent pleurectomy, 253 (32\%) pneumonectomy, 206 (27\%) surgical biopsy, and $48(6 \%)$ had other or unknown procedures. Data on pre- and post-operative chemotherapy was available in $522(67 \%)$ and $466(60 \%)$ cases, respectively. Pre-operative chemotherapy was given in 155 (30\%) cases, and post-operative chemotherapy in 222 $(48 \%)$ cases. Patients receiving post-op chemotherapy were significantly younger than those who did not receive post-op chemotherapy (61.3 vs 64.6 years, $P=0.0006)$. Cases differed by institution with respect to age $(P<0.0001)$, use of post-operative chemotherapy $(P<0.001)$, nuclear grade $(P<0.001)$, and necrosis $(P<0.001)$. There was no significant difference in gender by institution $(P=0.51)$. T stage and $\mathrm{N}$ status were not analyzed as these data were incomplete.
Table 1 Number of patients from each institution

\begin{tabular}{lr}
\hline Institution & Number \\
\hline Memorial Sloan Kettering Cancer Center & 170 \\
Basildon \& Thurrock University Hospital & 119 \\
Barts Health NHS Trust & 84 \\
University Hospital Zurich & 74 \\
Duke University Medical Center & 72 \\
Centre Léon Bérard & 60 \\
University of Chicago Medical Center & 48 \\
University of Leicester & 34 \\
Medical University of Graz & 28 \\
Cedars-Sinai Medical Center & 24 \\
Royal Brompton and Harefield Hospitals & 22 \\
Tokyo Women's Medical University Yachiyo Medical & 12 \\
Center & 11 \\
Fukuoka University & 7 \\
University Hospital of Wales & \\
University of Pittsburgh Medical Center and VA & \\
Pittsburgh & 7 \\
Healthcare System & 3 \\
University of Pennsylvania Perelman School of & \\
Medicine & 1 \\
University of Vermont Medical Center &
\end{tabular}

Scores for nuclear atypia and mitotic count were available in $546(70 \%)$ cases, as few institutions only provided the composite nuclear grade. A nuclear atypia score of 1 was present in $82(15 \%)$ cases, nuclear atypia score of 2 in $310(57 \%)$ cases, and nuclear atypia score of 3 in $154(28 \%)$ cases. A mitotic count score of 1 was found in 223 cases (41\%), a mitotic count score of 2 in 150 cases $(27 \%)$, and a mitotic count score of 3 in 173 (32\%) cases. Nuclear grade I cases had a frequency of 301 (39\%), nuclear grade II of 354 (45\%), and nuclear grade III of $121(16 \%)$. Necrosis was present in 235 (30\%) cases. Data on growth pattern were available in 708 (91\%) cases, of which 275 (39\%) had a predominately solid growth pattern.

The overall survival was 16 months (95\% Confidence interval (CI): $15.0-17.5)$. On univariate analysis, male gender $(P=0.0029)$, and age $(P=0.0091)$ were significantly associated with overall survival. The mortality rate was $33 \%$ higher (hazard ratio $(\mathrm{HR})=1.33$, 95\% CI: $1.10-1.60$ ) in males compared with females, with an increase of $15 \%$ per each 10-year increase in age (95\% CI: $1.07-1.24)$. Survival rates varied significantly by institution $(P<0.0001)$ with median overall survival ranging from 12 to 26 months.

Of the histologic features evaluated, nuclear grade, necrosis, growth pattern, nuclear atypia score, and mitotic count score were associated with overall survival upon univariate analysis (Table 2). Patients with nuclear grade III tumors had the worst median overall survival (8 months) followed by nuclear grade II (14 months) and nuclear grade I tumors (27 months, $P<0.0001$ ) (Figure 3a). In pairwise comparisons, overall survival was significantly different between patients with nuclear grade II and grade I tumors (HR 1.87, 95\% CI: 1.58-2.21, $P<0.0001)$, nuclear grade III and grade I tumors 
Table 2 Univariate analysis in predicting overall survival

\begin{tabular}{|c|c|c|c|}
\hline Variable & Patients (\%) & $\begin{array}{c}\text { Median } \\
\text { overall } \\
\text { survival } \\
\text { (months) }\end{array}$ & $\mathrm{P}$-value \\
\hline Age (years) & & & 0.009 \\
\hline$<40$ & $13(2)$ & 29 & $\begin{array}{l}\text { (per 10-year } \\
\text { increase in } \\
\text { age) }\end{array}$ \\
\hline $41-50$ & $54(7)$ & 17 & \\
\hline $51-60$ & $164(21)$ & 19 & \\
\hline $61-70$ & $289(37)$ & 16 & \\
\hline$>70$ & $256(33)$ & 9.3 & \\
\hline Sex & & & 0.0029 \\
\hline Male & $611(79)$ & 16 & \\
\hline Female & $165(21)$ & 19 & \\
\hline Procedure & & & 0.0001 \\
\hline Biopsy & $206(27)$ & 21 & $\begin{array}{l}\text { (Pl \& Pn vs } \\
\text { biopsy \& } \\
\text { other) }\end{array}$ \\
\hline Pleurectomy (Pl) & $269(35)$ & 17 & 0.022 \\
\hline $\begin{array}{l}\text { Pneunomectomy } \\
\text { (Pn) }\end{array}$ & $253(32)$ & 21 & (Pl vs $\mathrm{Pn}$ ) \\
\hline Other/unknown & $48(6)$ & 11 & \\
\hline Necrosis & & & 0.000 \\
\hline Present & $235(30)$ & 10 & \\
\hline Absent & $541(70)$ & 19 & \\
\hline $\begin{array}{l}\text { Solid growth pattern } \\
(>50 \%)\end{array}$ & & & 0.001 \\
\hline Yes & 275 (39) & 12 & \\
\hline No & $433(61)$ & 19 & \\
\hline Nuclear atypia & & & $\leq 0.009$ \\
\hline Score 1 & $82(15)$ & 31 & $\begin{array}{c}(1 \text { vs } 2-3,2 \\
\text { vs } 3)\end{array}$ \\
\hline Score 2 & $310(57)$ & 18 & \\
\hline Score 3 & $154(28)$ & 11 & \\
\hline Mitotic count & & & $\leq 0.011$ \\
\hline Score 1 & $223(41)$ & 22 & $\begin{array}{c}(1 \text { vs } 2-3,2 \\
\text { vs } 3)\end{array}$ \\
\hline Score 2 & $150(27)$ & 18 & \\
\hline Score 3 & $173(32)$ & 11 & \\
\hline Nuclear grade & & & $<0.000$ \\
\hline I & $301(39)$ & 27 & $\begin{array}{c}\text { (I vs II-III, II } \\
\text { vs III) }\end{array}$ \\
\hline II & $354(45)$ & 14 & \\
\hline III & $121(16)$ & 8 & \\
\hline $\begin{array}{l}\text { Mitosis-Necrosis } \\
\text { score }\end{array}$ & & & $\leq 0.020$ \\
\hline Score 0 & $299(55)$ & 22 & $\begin{array}{l}\text { (0 vs I-II, II } \\
\text { vs III) }\end{array}$ \\
\hline Score I & $149(27)$ & 14 & \\
\hline Score II & $98(18)$ & 10 & \\
\hline
\end{tabular}

(HR 3.40, 95\% CI 2.71-4.27, $P<0.0001$ ) and nuclear grade III and grade II tumors (HR 1.82, CI $1.47-2.26, P<0.0001)$. Of note, both components of nuclear grade (nuclear atypia and mitotic count) contributed independently to overall survival $(P<0.001)$. There was no evidence of an interaction between the two components $(P=0.56)$. Median overall survival was 22, 18, and 11 months among patients with mitotic count scores of 1,2 , and 3 , respectively; and 31,18 , and 11 months among patients with nuclear atypia scores of 1,2, and 3, respectively (Figures $3 \mathrm{~b}$ and $\mathrm{c}$ ).

Patients with tumors with necrosis had a significantly lower median overall survival than those without (10 vs 19 months, HR 1.85, CI: $1.57-2.17$, $P<0.0001$ ) (Figure 4a). Median overall survival was significantly lower patients with predominately solid growth pattern tumors (12 vs 19 months, HR 1.45, CI: $1.23-1.7, P<0.0001$ ) (Figure $4 \mathrm{~b}$ ). Independent from nuclear grade, there were no significant differences in overall survival between patients with a predominantly solid tumor compared to those with predominantly non-solid tumors $(P=0.44, P=0.84$, and $P=0.25$ within nuclear grades I, II, and III, respectively)

We next considered the utility of combining nuclear grade and necrosis to better stratify patients. In this analysis, the presence of necrosis within nuclear grade I and grade II tumors further separated patients by overall survival. A statistically significant difference in median overall survival was found between patients with nuclear grade II tumors with and without necrosis (10 vs 16 months, $\mathrm{HR}=1.42$, $95 \%$ CI: $1.13-1.78, P=0.002)$. Patients with nuclear grade I tumors with necrosis had a marginally significant lower median overall survival than those with grade I tumors without necrosis (16 vs 29 months, HR: 1.51, 95\% CI: 0.96-2.39, $P=0.076$ ). In nuclear grade III tumors, patients with necrosis fared better, but this did not reach statistical significance $\quad(\mathrm{HR}=0.70, \quad 95 \% \quad \mathrm{CI}: 0.47-1.03$, $P=0.073)$. Patients with nuclear grade I tumors with necrosis were associated with a similar overall survival to those with nuclear grade II tumors without necrosis (15.8 vs 16.3 months, 95\% CI $-0.33-0.58, P=0.60$ ). Patients with nuclear grade III tumors showed a significantly lower overall survival than those with nuclear grade II tumors with necrosis $(P=0.004)$. Overall, survival decreased significantly from patients with nuclear grade I tumors without necrosis (29 months) to those with nuclear grade I tumors with necrosis or nuclear grade II tumors without necrosis (16 months) to nuclear grade II tumors with necrosis (10 months), and nuclear grade III tumors (8 months) (Figure 5a).

Survival rates differed significantly according to the type of surgical procedure performed $(P<0.001)$ with patients undergoing pneumonectomy faring better than those undergoing pleurectomy (HR = $0.80,95 \%$ CI: $0.67-0.97, P=0.022$ ). In addition, patients undergoing pleurectomy or pneumonectomy had a significantly better overall survival compared with those who underwent biopsy.

In multivariate analysis, nuclear grade, necrosis, age, and sex were all significantly associated with overall survival $(P<0.001, P=0.030, P=0.006$, and $P=0.015$, respectively) (Table 3 ). The interaction between nuclear grade and necrosis described above 


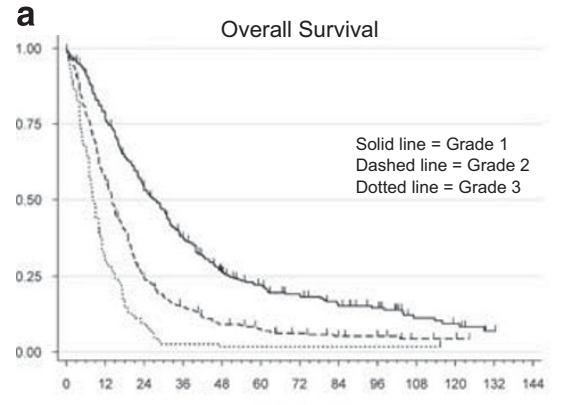

Time (months)

\begin{tabular}{|c|c|}
\hline Grade & Median OS \\
\hline Grade I & 27 months \\
\hline Grade II & 14 months \\
\hline Grade III & 8 months \\
\hline
\end{tabular}

b

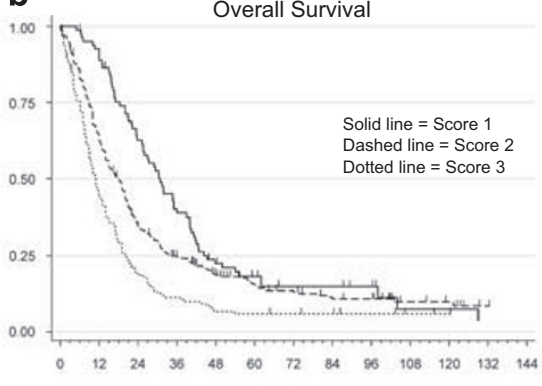

Time (months)

\begin{tabular}{|c|l|}
\hline Nuclear Score & Median OS \\
\hline Score 1 & 31 months \\
\hline Score 2 & 18 months \\
\hline Score 3 & 11 months \\
\hline
\end{tabular}

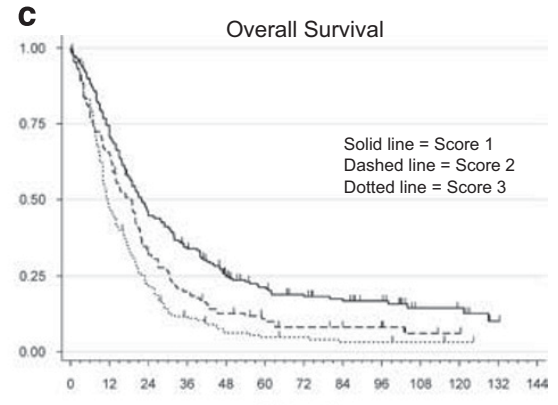

Time (months)

\begin{tabular}{|c|c|}
\hline Mitotic Score & Median OS \\
\hline Score 1 & 22 months \\
\hline Score 2 & 18 months \\
\hline Score 3 & 11 months \\
\hline
\end{tabular}

Figure 3 Overall survival by nuclear grade and its components. (a) Patients with nuclear grade I tumors had the best overall survival, followed by nuclear grade II and grade III. (b) Patients with a nuclear score of 1 had the best overall survival followed by scores of 2 and 3. (c) Patients with a mitotic score of 1 had the best overall survival, followed by scores 2 and 3.

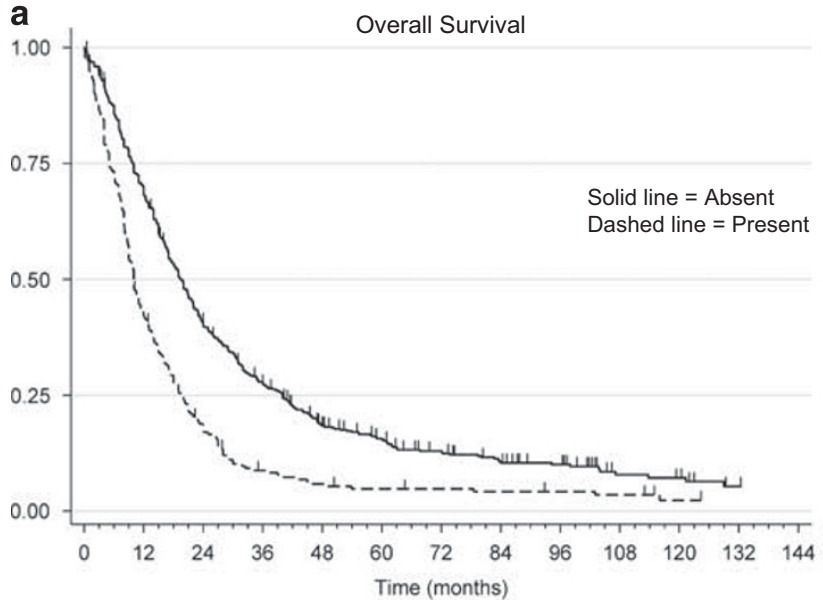

\begin{tabular}{|c|c|}
\hline Necrosis & Median OS \\
\hline Absent & 19 months \\
\hline Present & 10 months \\
\hline
\end{tabular}

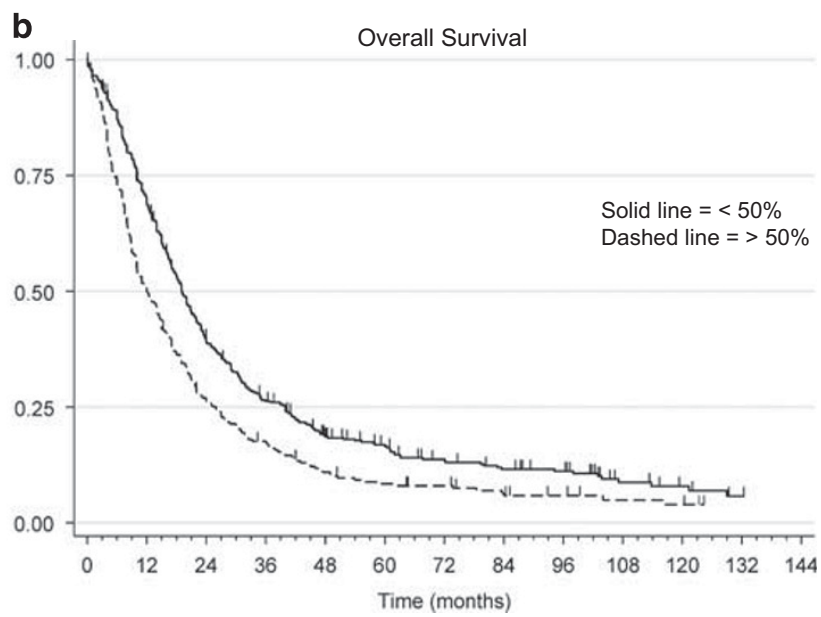

\begin{tabular}{|c|c|}
\hline$\%$ Solid growth & Median OS \\
\hline$<50 \%$ & 19 months \\
\hline$>50 \%$ & 12 months \\
\hline
\end{tabular}

Figure 4 Overall survival by necrosis and growth pattern. (a) Patients with tumors showing necrosis had a worse overall survival than those without necrosis. (b) Patients with tumors with a predominately solid growth pattern ( $>50 \%$ solid) had a worse overall survival than those with a predominately non-solid growth pattern on univariate analysis.

remains statistically significant after adjustment for age and sex. A predominantly solid growth pattern and surgical procedure were not independently associated with survival, however, after controlling for nuclear grade, necrosis, age, and sex. Some institutional differences remain after adjustment for age and sex, and the nuclear grade by necrosis interaction remains statistically significant. After controlling for nuclear grade, age and sex, the administration of post-operative chemotherapy was significantly associated with improved survival (HR $=0.57,95 \%$ CI: $0.46-0.69, P<0.001$ ), but preop chemotherapy was not (HR $=0.87,95 \%$ CI: $0.69-$ 1.07, $P=0.18)$.
There was a strong association between nuclear grade and the mitosis-necrosis score $(P<0.001)$ (Table 4). Patients with tumors having a mitosisnecrosis score of 0 had an overall survival of 22 months $(n=299)$, mitosis-necrosis score of I of 14 months $(n=149)$, a score of II of 10 months $(n=98)$. Pairwise comparisons between the three groups were all statistically significant: 0 versus I (HR:1.71, 95\% CI: 1.39-2.12, $P<0.001$ ), 0 versus II (HR: 2.35, 95\% CI 1.84-3.00, $P<0.001$ ), and I versus II (HR 1.37, 95\% CI 1.05-1.78, $P=0.020$ ) (Figure 5b). Note, however, that the three category mitosis-necrosis score does not give quite the same degree of separation in the survival curves 


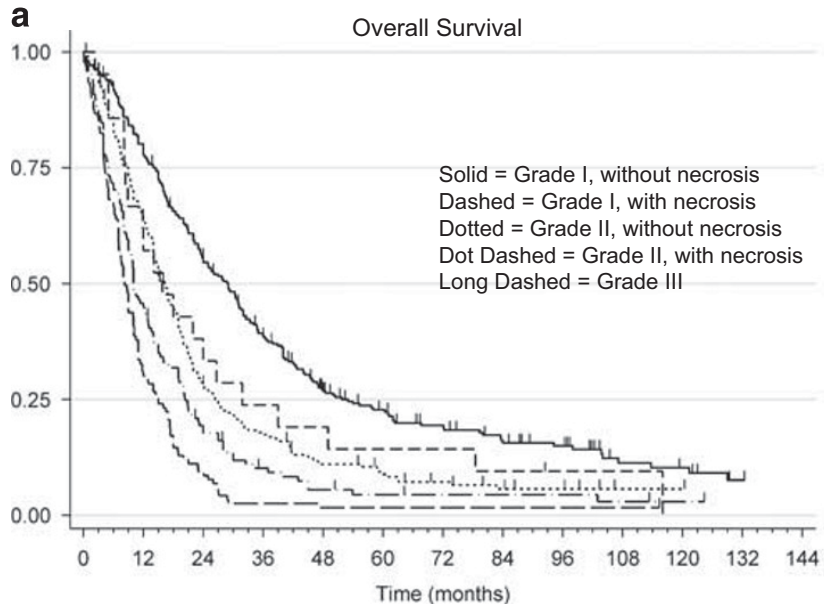

\begin{tabular}{|c|c|}
\hline Grade \& Necrosis & Median OS \\
\hline Grade I, without necrosis & 29 months \\
\hline Grade I, with necrosis & 16 months \\
\hline Grade II, without necrosis & 16 months \\
\hline Grade II, with necrosis & 10 months \\
\hline Grade III & 8 months \\
\hline
\end{tabular}

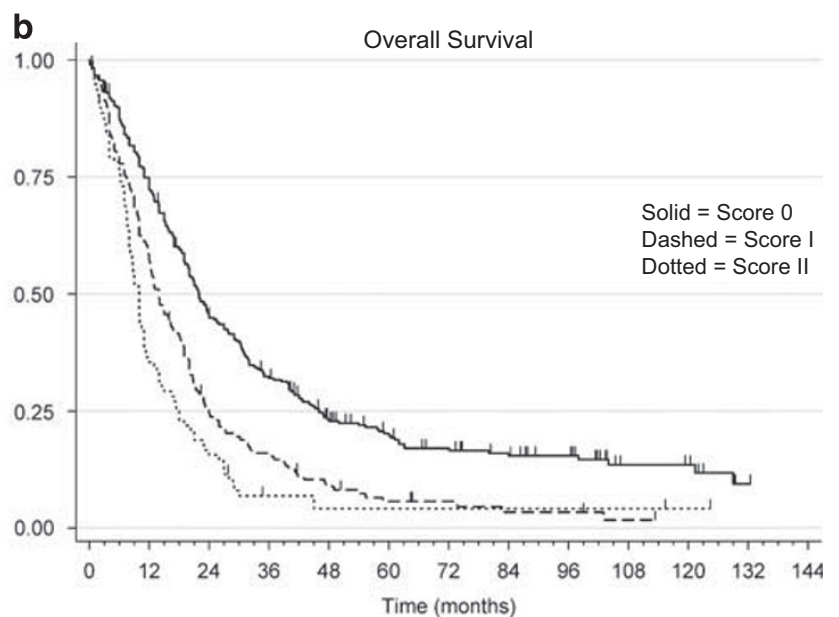

\begin{tabular}{|c|c|}
\hline Mitosis - Necrosis Score & Median OS \\
\hline Score 0 & 22 months \\
\hline Score I & 14 months \\
\hline Score II & 10 months \\
\hline
\end{tabular}

Figure 5 Overall survival by nuclear grade and necrosis, and mitosis-necrosis score. (a) Patients with nuclear grade I tumors without necrosis had the best overall survival. Patients with nuclear grade I tumors with necrosis and nuclear grade II tumors without necrosis had similar overall survival. Patients with nuclear grade II tumors with necrosis and nuclear grade III tumors had the worst overall survival. (b) Patients with tumors with a mitosis-necrosis score of 0 had a better overall survival than patients with scores of I and II.

Table 3 Multivariate analysis in predicting overall survival

\begin{tabular}{lccc}
\hline Variables & Hazard ratio & $95 \%$ CI & P-value \\
\hline Nuclear atypia & & & \\
$\quad$ Score 1 vs 2 & 1.42 & $1.09-1.85$ & 0.009 \\
$\quad$ Score 1 vs 3 & 2.26 & $1.69-3.01$ & $<0.0001$ \\
& & & \\
Mitotic count & 1.45 & $1.16-1.82$ & 0.001 \\
$\quad$ Score 1 vs 2 & 1.96 & $1.58-2.43$ & $<0.0001$ \\
$\quad$ Score 1 vs 3 & & & \\
& & & \\
Nuclear grade & 1.76 & $1.47-2.10$ & $<0.0001$ \\
$\quad$ Grade I vs II & 2.90 & $2.23-3.77$ & $<0.0001$ \\
$\quad$ Grade I vs III & & & \\
Mitosis-necrosis score & 1.72 & $1.39-2.12$ & $<0.0001$ \\
$\quad$ Score I vs II & 2.35 & $1.84-3.00$ & $<0.0001$ \\
$\quad$ Score I vs III & & & \\
$\quad$ Necrosis & 1.23 & $1.02-1.48$ & 0.030 \\
$\quad$ Present vs absent & & & \\
$\quad$ Age & & & \\
$\quad$ Per decade increase in & 1.01 & $1.00-1.02$ & 0.006 \\
age & & & \\
Sex & & & \\
$\quad$ Male vs female & 1.27 & $1.05-1.53$ & 0.015 \\
\hline
\end{tabular}

CI, confidence interval.
Table 4 Association between nuclear grade and mitosis-necrosis $(\mathrm{M}-\mathrm{N})$ score

\begin{tabular}{lcccc}
\hline & & \multicolumn{4}{c}{ Mitosis-necrosis score } \\
\cline { 3 - 5 } Nuclear grade & No. of patients (\%) & 0 & 1 & 2 \\
\hline I & 202 & $187(93)^{\mathrm{a}}$ & $15(7)$ & $0(0)$ \\
II & 269 & $112(43)$ & $115(43)$ & $41(15)$ \\
III & 76 & $0(0)$ & $19(25)$ & $57(75)$ \\
\hline
\end{tabular}

${ }^{\mathrm{a}}$ Row percent.

as the five category nuclear grade/necrosis combination.

Finally, to assess whether the stratification of patients according to nuclear grade and necrosis was robust across institutions, we applied this classification among the three institutions contributing the highest numbers of patients: Memorial Sloan Kettering Cancer Center $(n=170)$, Basildon \& Thurrock University Hospital $(n=119)$, and Barts Heath NHS Trust $(n=84)$. For each clinical center, the stratification according to nuclear grade and necrosis led to distinctly prognostic subsets $(P<0.001$, $P<0.001$, and $P=0.002$, respectively). 


\section{Discussion}

This multi-institutional and multiple pathologist study clearly demonstrates the value of nuclear grade and necrosis in predicting overall survival in epithelioid malignant pleural mesothelioma. Current clinical parameters are poor predictors of overall survival. When dividing epithelioid malignant pleural mesothelioma into low and high stage, Kadota et $a l^{6}$ found that this differentiates overall survival at best by 15 vs 20 months, with a distribution of 60 versus $40 \%$. Kadota et al ${ }^{6}$ sought to determine histopathological prognostic factors by studying 232 epithelioid malignant pleural mesothelioma and discovered that nuclear atypia and mitotic count were independently associated with overall survival. Habougit et al, ${ }^{14}$ in a study of 77 epithelioid malignant pleural mesothelioma, also showed that nuclear atypia and mitotic count were predictive of overall survival, in addition to atypical mitoses, nuclear atypia, prominent nucleoli, and necrosis.

We found that nuclear grading stratifies patients into three distinct groups with median survival times of 27 vs 14 vs 3 months. Our results are consistent with those of Kadota et $a l^{6}$ who found a median overall survival between nuclear grade groups of 28 vs 14 vs 5 months. In addition, Kadota et $a l^{6}$ found that the nuclear grading system remained significant in sub-cohorts of low and high stage patients and was also predictive of time to recurrence.

While Kadota et al examined cases from a single institution, our study includes a larger cohort of patients from multiple institutions, in which histologic examination was performed by multiple pathologists. Even though cases differed by institution with respect to age, use of post-operative chemotherapy, nuclear grade, and necrosis, after multivariate analysis nuclear grade remained predictive of overall survival despite these differences. As such, these differences in the presence of necrosis are likely due to differences in patient population as suggested by the difference in age and less likely to be due to interobserver variability. The issue of interobserver variability has been raised in nuclear grading and mitotic count, however these concerns are mitigated by using welldefined criteria, which were applied in this study. ${ }^{15-18}$

We examined two additional histologic features, growth pattern and necrosis to determine if they were prognostic. A predominantly solid growth pattern has been associated with a poor outcome in epithelioid malignant pleural mesothelioma, with a median overall survival of 13.7 months versus 16.3 months for all comers. ${ }^{7}$ The presence of necrosis has also been shown to be a predictor of poor outcome in epithelioid malignant pleural mesothelioma. ${ }^{14}$ Although a predominantly solid growth pattern was associated with worse median overall survival in univariate analysis, it was not independently significant. The significance of solid growth on univariate analysis was likely related to the increasing incidence of predominantly solid growth with increasing nuclear grade. Necrosis was independently associated with overall survival and stratified patients into two groups with a survival of 10 vs 19 months. Our results mirror those of a recent study of 77 epithelioid malignant pleural mesothelioma in which necrosis was also associated with a worse overall survival of 10 vs 19 months. ${ }^{14}$ When we examined the presence of necrosis within nuclear grade groups, we were able to further stratify median overall survival within nuclear grade I (16 vs 29) and nuclear grade II (10 vs 16) groups. As nuclear grade I tumors with necrosis behaved similarly to nuclear grade II tumors without necrosis (the median survival time for both was approximately 16 months), and those with necrosis did not fare worse than those without necrosis in nuclear grade III tumors, the combination of necrosis and nuclear grade allows classification of epithelioid malignant pleural mesothelioma into four distinct prognostic groups by median overall survival: nuclear grade I tumors without necrosis (29 months), nuclear grade I tumors with necrosis and nuclear grade II tumors without necrosis (16 months), nuclear grade II tumors with necrosis (10 months) and nuclear grade III tumors (8 months).

The majority of patients in the Kadota et al study underwent extrapleural pneumonectomy or pleurectomy $(89 \%)$ and fewer than $5 \%$ underwent biopsy, whereas the majority of patients in the Habougit et al study underwent biopsy (97\%). ${ }^{7,14}$ In our study, $68 \%$ of patients underwent pleurectomy or pneumonectomy and 27\% underwent biopsy. Although patients who underwent pneumonectomy had a better survival than those with pleurectomy and both of these categories had a better survival than those who received biopsy, the type of surgical procedure was not randomized. Therefore, differences in outcomes between patients who underwent pneumonectomy versus those who received pleurectomy or biopsy may be confounded by other factors. Also, as staging information was incomplete, it is possible that patients who received biopsy alone had more advanced disease than those who underwent resection.

In this study we propose an alternative scoring system, the mitosis-necrosis score, which avoids assessment of nuclear atypia. We sought to replace this more subjective component of the nuclear grading system with necrosis since we have shown this factor to be independently prognostic. In addition to necrosis being less subjective, it is also easy to assess. The mitosis-necrosis scoring system did stratify epithelioid malignant pleural mesothelioma into distinct groups by median overall survival (22 vs 14 vs 10 months), although this separation is not as strong as that provided by the combination of nuclear grade and necrosis.

In multivariate analysis, post-operative but not pre-operative chemotherapy was associated with improved survival. Despite this fact, nuclear grade and necrosis remained independently predictive of overall survival. Owing to the poor prognosis of epithelioid malignant pleural mesothelioma, a multimodality approach including surgery, chemotherapy, and radiation is often taken. In this approach, 
chemotherapy is more often administered in the neoadjuvant setting but can also be administered adjuvantly post-surgery or intracavitary with hyperthermia at the time of cytoreduction. ${ }^{3,19,20}$ There is a lack of randomized trials regarding outcome in patients treated with hyperthermic intraoperative chemotherapy, however patients with low-stage epithelioid malignant pleural mesothelioma receiving this therapy have been shown to have a longer survival and progression free interval. ${ }^{19,20}$

The strengths of the study include that it is multiinstitutional and involved multiple pathologists with a large number of cases. In addition, the largest contribution of cases was from Memorial Sloan Kettering Cancer Center and whereas the results were mostly similar to those found in a prior study from that institution, our additional cohorts appear to have validated those findings. ${ }^{6}$ Nuclear grading and assessment of necrosis can be performed easily on H\&E-stained sections. These factors can be incorporated into a mesothelioma synoptic to be included in the pathology report, and may help clinicians to decide on clinical management. The limitations include that the analysis was retrospective and that there was no analysis of interobserver variability in histological grading. In the future, the authors hope to apply the nuclear grade and mitosisnecrosis score prospectively to evaluate the predictive value of these systems. We will additionally extend the study to peritoneal malignant mesothelioma to determine if these grading systems are predictive of overall survival in this tumor.

In conclusion, nuclear grade and necrosis predict overall survival in epithelioid malignant pleural mesothelioma. The addition of necrosis to nuclear grade allows for greater separation of overall survival. An alternative scoring system is the mitosisnecrosis score, which while not as robust as nuclear grade and necrosis does stratify patients into distinct prognostic groups. These scoring systems can be applied to both resection and biopsy specimens.

\section{Disclosure/conflict of interest}

The authors declare no conflict of interest.

\section{References}

1 Kindler HL, Campbell NP. Update on malignant pleural mesothelioma. Semin Respir Crit Care Med 2011;32: 102-110.

2 Rusch VK, Giroux D, Kennedy C, et al. Initial analysis of the international association for the study of lung cancer mesothelioma database. J Thorac Oncol 2012;7: 1631-1639.

3 Van Schil PE, Opitz I, Weder W. Multimodal management of malignant pleural mesothelioma: where are we today? Eur Respir J 2014;44:754-764.
4 Zellos L, Christiani DC. Epidemiology, biologic behavior, and natural history of mesothelioma. Thorac Surg Clin 2014;14:469-477.

5 Taioli W, Wolf AS, Camacho-Rivera M. Determinants of survival in malignant pleural mesothelioma: a surveillance, epidemiology, and end results (SEER) study of 14,228 patients. PLOS ONE 2015;10:e0145039.

6 Kadota K, Suzuki K, Colovos C, et al. A nuclear grading system is a strong predictor of survival in epitheloid diffuse malignant pleural mesothelioma. Mod Pathol 2012;25:260-271.

7 Galateau-Salle F, Churg A, Roggli V. Tumours of the pleura. Mesothelial tumours. Diffuse malignant mesothelioma. Epithelioid Mesothelioma. In: Travis WD, Brambilla E, Burke AP, Marx A, Nicholson AG (eds). The World Health Organisation Classification of tumours of the lung, pleura, thymus and heart. 7th edn. International Agency of Research on Cancer: Lyon, France, 2015;156-164.

8 Kadota K, Suzuki K, Sima CS, Rusch VW, Adusumilli PS, Travis WD. Pleomorphic epithelioid diffuse malignant pleural mesothelioma. A clinicopathologic review and conceptual proposal to reclassify as biphasic or sarcomatoid mesothelioma. J Thorac Oncol 2011;6: 896-904.

9 Husain AN, Colby T, Ordonez N, et al. Guidelines for the pathologic diagnosis of malignant mesothelioma: 2012 update of the consensus statement from the international mesothelioma interest group. Arch Pathol Lab Med 2013;137:647-667.

10 Brookmeyer R, Crowley J. A confidence interval for the median survival time. Biometrics 1982;38:29-41.

11 Kaplan EL, Meier P. Nonparametric estimation from incomplete observations. J Am Stat Assoc 1958;53: 457-481.

12 Cox DR. Regression models and life tables (with discussion). J R Stat Soc Series B 1972;34:187-220.

13 Kay R. Proportional hazard regression models and the analysis of censored survival data. Appl Statist 1977;26:227-237.

14 Habougit C, Thrombert-Paviot B, Karpathiou G, et al. Histopathologic features predict survival in diffuse pleural malignant mesothelioma on pleural biopsies. Virchows Arch 2017;470:639-646.

15 Dalton LW, Pinder SE, Elston CE, et al. Histopathologic grading of breast cancer: linkage of patient outcome with level of pathologist agreement. Mod Pathol 2000;13: 730-735.

16 Cross SS. Grading and scoring in histopathology. Histopathology 1998;33:99-106.

17 Baak JP. Mitosis counting in tumors. Hum Pathol 1990;21: 683-685.

18 Thompson LD. Pheochromocytoma of the Adrenal gland Scaled Score (PASS) to separate benign from malignant neoplasms: a clinicopathologic and immunophenotypic study of 100 cases. Am J Surg Pathol 2002;26:551-566.

19 Kotova S, Wong RM, Cameron RB. New and emerging therapeutic options for malignant pleural mesothelioma: review of early clinical trials. Cancer Manag Res 2015;7:51-63.

20 Sugarbaker DJ, Gill RR, Yeap BY, et al. Hyperthermic intraoperative pleural cisplatin chemotherapy extends interval to recurrence and survival among low-risk patients with malignant pleural mesothelioma undergoing surgical macroscopic complete resection. J Thorac Cardiovasc Surg 2013;145:995-963. 